\title{
HARMLESS DELAYS IN A DISCRETE RATIO-DEPENDENT PERIODIC PREDATOR-PREY SYSTEM
}

\author{
YONG-HONG FAN AND WAN-TONG LI
}

Received 18 December 2005; Accepted 13 February 2006

Verifiable criteria are established for the existence of positive periodic solutions and permanence of a delayed discrete periodic predator-prey model with Holling-type II functional response $N_{1}(k+1)=N_{1}(k) \exp \left\{b_{1}(k)-a_{1}(k) N_{1}\left(k-\left[\tau_{1}\right]\right)-\alpha_{1}(k) N_{2}(k) /\left(N_{1}(k)+\right.\right.$ $\left.\left.m(k) N_{2}(k)\right)\right\}$ and $N_{2}(k+1)=N_{2}(k) \exp \left\{-b_{2}(k)+\alpha_{2}(k) N_{1}\left(k-\left[\tau_{2}\right]\right) /\left(N_{1}\left(k-\left[\tau_{2}\right]\right)+\right.\right.$ $\left.\left.m(k) N_{2}\left(k-\left[\tau_{2}\right]\right)\right)\right\}$. Our results show that the delays in the system are harmless for the existence of positive periodic solutions and permanence of the system. In particular our investigation confirms that if the death rate of the predator is rather small as well as the intrinsic growth rate of the prey is relatively large, then the species could coexist in the long run.

Copyright (c) 2006 Y.-H. Fan and W.-T. Li. This is an open access article distributed under the Creative Commons Attribution License, which permits unrestricted use, distribution, and reproduction in any medium, provided the original work is properly cited.

\section{Introduction}

In mathematical biology, the dynamics of the growth of a population can be described if the functional behavior of the rate of growth is known. It is this functional behavior which is usually measured in the laboratory or in the field. Among the relationships between the species living in the same outer environment, the predator-prey theory plays an important and fundamental role. The dynamic relationship between predators and their preys has long been and will continue to be one of the dominant themes in both ecology and mathematical ecology due to its universal existence and importance (Berryman [4]). These problems may appear to be simple mathematically at first sight, they are in fact very challenging and complicated. There are many different kinds of predator-prey models in the literature; for more details we can refer to $[4,7]$. In general, a predator-prey system takes the form

$$
\begin{gathered}
x^{\prime}=r x\left(1-\frac{x}{K}\right)-\varphi(x) y, \\
y^{\prime}=y(\mu \varphi(x)-D),
\end{gathered}
$$

Hindawi Publishing Corporation Discrete Dynamics in Nature and Society Volume 2006, Article ID 12176, Pages 1-21 DOI 10.1155/DDNS/2006/12176 
where $\varphi(x)$ is the functional response function, which reflects the capture ability of the predator to prey. For more biological meaning, the reader may consult $[7,19]$. Massive work has been done on this issue. We refer to the monographs [8, 16, 21, 24] for general delayed biological systems and to $[18,22,23,25,26,28,29]$ for investigations on predator-prey systems.

Until very recently, both ecologists and mathematicians chose to base their studies on this traditional prey-dependent functional response predator-prey system which is called prey-dependent model [12]. But there is a growing explicit biological and physiological evidence $[3,11,14,17]$ that in many situations, especially when predators have to search for food (and, therefore, have to share or compete for food), a more suitable general predator-prey theory should be based on the so-called ratio-dependent theory, which can be roughly stated as that the per capita predator growth rate should be a function of the ratio of prey to predator abundance, and so should be the so-called ratio-dependent functional response. This is strongly supported by numerous field and laboratory experiments and observations $[2,9]$. A general form of a ratio-dependent model is

$$
\begin{gathered}
x^{\prime}=r x\left(1-\frac{x}{K}\right)-\varphi\left(\frac{x}{y}\right) y, \\
y^{\prime}=y\left(\mu \varphi\left(\frac{x}{y}\right)-D\right) .
\end{gathered}
$$

Here the predator-prey interactions are described by $\varphi(x / y)$ instead of $\varphi(x)$ in (1.1). This can be interpreted as when the numbers of predators change slowly (relative to the change of their prey), there is often competition among the predators, and the per capita rate of predation depends on the numbers of both prey and predator, most likely and simply on their ratio. For the system (1.2) with periodic coefficients, in [5] we explored the existence of periodic solutions with delays. In addition, most research works concentrate on the socalled Michaelis-Menten-type ratio-dependent predator-prey model:

$$
\begin{gathered}
x^{\prime}=r x\left(1-\frac{x}{K}\right)-\frac{\alpha x y}{m y+x}, \\
y^{\prime}=y\left(-d+\frac{f x}{m y+x}\right)
\end{gathered}
$$

see $[3,11,14,17,27]$ and references therein. The functional response function $\varphi(u)=$ $c u /(m+u), u=x / y$, in the above model was used by Holling [10] as Holling-type II functional response, it usually describes the uptake of substrate by the microorganisms in microbial dynamics or chemical kinetics [7].

On the other hand, though most predator-prey theories are based on continuous models governed by differential equations, the discrete time models are more appropriate than the continuous ones when the size of the population is rarely small or the population has nonoverlapping generations $[1,21]$. And in ecosystems, an important theme that interested mathematicians as well as biologists is whether the species in these systems would survive in the long run. That is, whether the ecosystems are permanent. As far as we know, 
few investigations have been carried out for the permanence on delayed discrete ecological systems since the dynamics of these systems are usually more complicated than the continuous ones. Just as pointed out in [8], even if the coefficients are constants, the asymptotic behavior of the discrete system is rather complex and "chaotic" than the continuous one. For example, consider the logistic equation

$$
x^{\prime}(t)=r x(t)\left[1-\frac{x(t)}{K}\right], \quad t \geq 0
$$

where $r$ and $K$ are both positive constants, and its corresponding discrete equation

$$
x(n+1)=x(n) \exp \left\{r\left[1-\frac{x(n)}{K}\right]\right\}, \quad n=0,1,2, \ldots
$$

It is known from the works of May [20] that for certain parameter values of $r$, the asymptotic behavior of the solutions of (1.5) is complex and "chaotic." While the solutions of (1.4) are normal.

Now we introduce some notations and definitions for the sake of convenience. Denote $\mathbb{Z}, \mathbb{R}$, and $\mathbb{R}^{+}$as the sets of all integers, real numbers, and nonnegative real numbers, respectively. Let $C$ denote the set of all bounded sequence $f: \mathbb{Z} \rightarrow \mathbb{R}, C_{+}$the set of all $f \in C$ such that $f>0$, and $C_{\omega}=\left\{f \in C_{+} \mid f(k+\omega)=f(k), k \in \mathbb{Z}\right\}, I_{\omega}=\{0,1, \ldots, \omega-1\}$. We also define

$$
f^{M}=\sup _{k \in I_{\omega}} f(k), \quad f^{L}=\inf _{k \in I_{\omega}} f(k), \quad \bar{f}=\frac{1}{\omega} \sum_{k=0}^{\omega-1} f(k)
$$

for any $\omega$-periodic sequence $\{f(k)\}$, where $k \in \mathbb{Z}$.

In view of periodicity of the actual environment, we begin with the periodic continuous ratio-dependent predator-prey system with Holling-type II functional response:

$$
\begin{gathered}
\frac{d N_{1}(t)}{d t}=N_{1}(t)\left[b_{1}(t)-a_{1}(t) N_{1}\left(t-\tau_{1}\right)\right]-\frac{\alpha_{1}(t) N_{1}(t) N_{2}(t)}{N_{1}(t)+m(t) N_{2}(t)}, \\
\frac{d N_{2}(t)}{d t}=N_{2}(t)\left[-b_{2}(t)+\frac{\alpha_{2}(t) N_{1}\left(t-\tau_{2}\right)}{N_{1}\left(t-\tau_{2}\right)+m(t) N_{2}\left(t-\tau_{2}\right)}\right],
\end{gathered}
$$

where $N_{1}(t)$ and $N_{2}(t)$ represent the densities of the prey population and predator population at time $t$, respectively; $\tau_{1} \geq 0$ and $\tau_{2} \geq 0$ are real constants; $b_{i}: \mathbb{R} \rightarrow \mathbb{R}$ and $m, a_{1}, \alpha_{i}$ : $\mathbb{R} \rightarrow \mathbb{R}^{+}(i=1,2)$ are continuous periodic functions with period $\omega>0$ and $\int_{0}^{\omega} b_{i}(t) d t>0$ $(i=1,2) ; b_{1}(t)$ stands for prey intrinsic growth rate, $b_{2}(t)$ stands for the death rate of the predator, $\alpha_{1}(t)$ and $\alpha_{2}(t)$ stand for the conversion rates, $m(t)$ stands for half capturing saturation; the function $N_{1}(t)\left[b_{1}(t)-a_{1}(t) N_{1}\left(t-\tau_{1}\right)\right]$ represents the specific growth rate of the prey in the absence of predator; and $N_{1}(t) /\left(N_{1}(t)+m(t) N_{2}(t)\right)$ denotes the ratiodependent response function, which reflects the capture ability of the predator. Similar 
4 Harmless delays in a discrete periodic model

to the arguments of [6], we can obtain a discrete time analogue of (1.7):

$$
\begin{gathered}
N_{1}(k+1)=N_{1}(k) \exp \left\{b_{1}(k)-a_{1}(k) N_{1}\left(k-\left[\tau_{1}\right]\right)-\frac{\alpha_{1}(k) N_{2}(k)}{N_{1}(k)+m(k) N_{2}(k)}\right\}, \\
N_{2}(k+1)=N_{2}(k) \exp \left\{-b_{2}(k)+\frac{\alpha_{2}(k) N_{1}\left(k-\left[\tau_{2}\right]\right)}{N_{1}\left(k-\left[\tau_{2}\right]\right)+m(k) N_{2}\left(k-\left[\tau_{2}\right]\right)}\right\},
\end{gathered}
$$

where $[t]$ denotes the integer part of $t>0$.

The exponential form of (1.8) assures that, for any initial condition $N(0)>0, N(k)$ remains positive. In the remainder of this paper, for biological reasons, we only consider solutions $N(k)$ with

$$
N_{i}(-k) \geq 0, \quad k=1,2, \ldots, \max \left\{\left[\tau_{1}\right],\left[\tau_{2}\right]\right\} ; \quad N_{i}(0)>0, \quad i=1,2 .
$$

If $\left[\tau_{1}\right]=\left[\tau_{2}\right]=0$, then system $(1.8)$ reduces to

$$
\begin{gathered}
N_{1}(k+1)=N_{1}(k) \exp \left\{b_{1}(k)-a_{1}(k) N_{1}(k)-\frac{\alpha_{1}(k) N_{2}(k)}{N_{1}(k)+m(k) N_{2}(k)}\right\}, \\
N_{2}(k+1)=N_{2}(k) \exp \left\{-b_{2}(k)+\frac{\alpha_{2}(k) N_{1}(k)}{N_{1}(k)+m(k) N_{2}(k)}\right\} .
\end{gathered}
$$

Recently, Fan and Wang [6] considered the existence of positive periodic solution for system (1.10) and obtained the following.

Theorem 1.1. Assume that the following conditions hold:

(H1) $\bar{b}_{1}>\overline{\left(\alpha_{1} / m\right)}$,

(H2) $\bar{\alpha}_{2}>\bar{b}_{2}$.

Then (1.10) has at least one positive $\omega$-periodic solution.

Huo and Li [13] further considered the permanent of system (1.10) and established the following result.

Theorem 1.2. Assume that

$$
b_{1}^{L}>\frac{\alpha_{1}^{M}}{m^{L}}, \quad \alpha_{2}^{L}>b_{2}^{M} .
$$

Then system (1.10) is permanent.

In this paper, our aim is to consider the effect of delays for the existence of positive periodic solutions and permanence of system (1.8). Our results show that delays in (1.8) are harmless for the existence of positive periodic solutions and permanence of (1.8). That is to say, we establish the following results.

Theorem 1.3. Assume that (H1) and (H2) hold. Then (1.8) has at least one positive wperiodic solution.

Since its proof is similar to that of [6], we omit it here.

Theorem 1.4. Assume that (H1) and (H2) hold. Then system (1.8) is permanent. 
Clearly, Theorem 1.3 extends Theorem 1.1; Theorem 1.4 extends and improves Theorem 1.2 by weaker conditions $(\mathrm{H} 1)$ and $(\mathrm{H} 2)$ instead of (1.11). In particular our investigation confirms that if the death rate of the predator is rather small as well as the intrinsic growth rate of the prey is relatively large, then the species could coexist in the long run.

\section{Proof of Theorem 1.4}

In this section, we prove Theorem 1.4. Before proving our main result, we list the definition of permanence and prove a lemma.

Definition 2.1. System (1.8) is said to be permanent if there exists two positive constants $\lambda_{1}$ and $\lambda_{2}$ such that

$$
\lambda_{1} \leq \liminf _{k \rightarrow \infty} N_{i}(k) \leq \limsup _{k \rightarrow \infty} N_{i}(k) \leq \lambda_{2}, \quad i=1,2,
$$

for any solution $\left(N_{1}(k), N_{2}(k)\right)$ of (1.8).

The following lemma will be useful to establish the main result.

Lemma 2.2. The problem

$$
\begin{gathered}
x(k+1)=x(k) \exp \{a(k)-b(k) x(k)\}, \\
x(0)=x_{0}>0,
\end{gathered}
$$

has at least one periodic solution $U$ if $b \in C_{\omega}, a \in C$, and $a$ is an w-periodic sequence with $\bar{a}>0$; moreover, the following properties hold:

(a) $U$ is positive $\omega$-periodic;

(b) U has the following estimations for its boundary:

$$
\frac{\bar{a}}{\bar{b}} \exp \{-(\overline{|a|}+\bar{a}) \omega\} \leq U(k) \leq \frac{\bar{a}}{\bar{b}} \exp \{(\overline{|a|}+\bar{a}) \omega\}
$$

especially,

$$
\frac{\bar{a}}{\bar{b}} \exp \{-\bar{a} \omega\} \leq U(k) \leq \frac{\bar{a}}{\bar{b}} \exp \{\bar{a} \omega\}
$$

if $a \in C_{\omega}$.

Proof. First, we prove (a). Notice that in (1.8), let $\alpha_{1}(k) \equiv 0, \tau_{1}=0$, then (1.8) can be reduced to

$$
\begin{aligned}
& N_{1}(k+1)=N_{1}(k) \exp \left\{b_{1}(k)-a_{1}(k) N_{1}(k)\right\}, \\
& N_{2}(k+1)=N_{2}(k) \exp \left\{-b_{2}(k)+\frac{\alpha_{2}(k) N_{1}\left(k-\left[\tau_{2}\right]\right)}{N_{1}\left(k-\left[\tau_{2}\right]\right)+m(k) N_{2}\left(k-\left[\tau_{2}\right]\right)}\right\},
\end{aligned}
$$


6 Harmless delays in a discrete periodic model

and the condition $(\mathrm{H} 1)$ of Theorem 1.1 reduces to $\bar{b}_{1}>0$. By Theorem 1.3, (2.5) has at least one positive $\omega$-periodic solution provided that $\bar{b}_{1}>0$ and $\bar{\alpha}_{2}>\bar{b}_{2}$. This implies that

$$
N_{1}(k+1)=N_{1}(k) \exp \left\{b_{1}(k)-a_{1}(k) N_{1}(k)\right\}
$$

has at least one positive $\omega$-periodic solution under the assumptions $\bar{b}_{1}>0$. That is to say,

$$
\begin{gathered}
x(k+1)=x(k) \exp \{a(k)-b(k) x(k)\}, \\
x(0)=x_{0}>0
\end{gathered}
$$

has at least one positive $\omega$-periodic solution provided that $\bar{a}>0$. The proof of (a) is complete.

The first part of (b) can be proved by the same method as that in [6], we only need to prove the second part of (b). In view of (a), set $U(k)=\exp \{z(k)\}$, then

$$
z(k+1)-z(k)=a(k)-b(k) \exp \{z(k)\}
$$

thus

$$
0=\sum_{k=0}^{\omega-1}(z(k+1)-z(k))=\bar{a} \omega-\sum_{k=0}^{\omega-1} b(k) \exp \{z(k)\}
$$

this implies

$$
(z(k))^{L} \leq \ln \left(\begin{array}{l}
\bar{a} \\
\bar{b}
\end{array}\right) \leq(z(k))^{M}
$$

Denote $z(\xi)=(z(k))^{L}$, where $\xi \in I_{\omega}$. By (2.8), $z(k+1)-z(k) \leq a(k)$, then for any $k \in I_{\omega}$ and $k \geq \xi$, we have

$$
\sum_{i=\xi}^{k}(z(i+1)-z(i)) \leq \sum_{i=\xi}^{k} a(i)
$$

since $a \in C_{\omega}$,

$$
\sum_{i=\xi}^{k}(z(i+1)-z(i)) \leq \sum_{i=\xi}^{k} a(i) \leq \bar{a} \omega,
$$

this shows that

$$
z(k+1) \leq z(\xi)+\bar{a} \omega, \quad \text { for } k \in I_{\omega}, k \geq \xi
$$

On the other hand,

$$
z(k+1)-z(k)=a(k)-b(k) \exp \{z(k)\} \geq-b(k) \exp \{z(k)\}
$$


hence

$$
\begin{aligned}
\sum_{i=k}^{\xi-1}(z(i+1)-z(i)) & \geq-\sum_{i=k}^{\xi-1} b(i) \exp \{z(i)\} \\
& \geq-\sum_{i=0}^{\omega-1} b(i) \exp \{z(i)\}=-\bar{a} \omega, \quad \text { for } k \in I_{\omega}, k \leq \xi-1
\end{aligned}
$$

therefore

$$
z(k) \leq z(\xi)+\bar{a} \omega, \quad \text { for } k \in I_{\omega}, k \leq \xi-1 .
$$

By (2.13) and (2.16), we can obtain $z(k) \leq z(\xi)+\bar{a} \omega$, for $k \in I_{\omega}$; thus

$$
U(k)=\exp \{z(k)\} \leq \frac{\bar{a}}{\bar{b}} \exp \{\bar{a} \omega\}
$$

by a similar analysis as above, we can obtain

$$
U(k) \geq \frac{\bar{a}}{\bar{b}} \exp \{-\bar{a} \omega\}
$$

This completes the proof of the second part of (b).

To prove Theorem 1.4, we need the following several propositions. For the rest of this paper, we consider the solution of (1.8) with initial conditions (1.9). For the definition of semicycle and related concepts, we refer to [15].

Proposition 2.3. There exists a positive constant $K_{1}$ such that $\lim _{\sup _{k \rightarrow+\infty}} N_{1}(k) \leq K_{1}$.

Proof. Given any positive solution $\left(N_{1}(k), N_{2}(k)\right)$ of (1.8), from the first equation of (1.8), we have

$$
N_{1}(k+1) \leq N_{1}(k) \exp \left\{b_{1}(k)-a_{1}(k) N_{1}\left(k-\left[\tau_{1}\right]\right)\right\}
$$

Let $N_{1}(k)=\exp \left\{u_{1}(k)\right\}$, then

$$
u_{1}(k+1)-u_{1}(k) \leq b_{1}(k)-a_{1}(k) \exp \left\{u_{1}\left(k-\left[\tau_{1}\right]\right)\right\} ;
$$

thus

$$
\sum_{i=k-\left[\tau_{1}\right]}^{k-1}\left(u_{1}(i+1)-u_{1}(i)\right) \leq \sum_{i=k-\left[\tau_{1}\right]}^{k-1} b_{1}(i)
$$

which is equivalent to

$$
u_{1}(k)-\sum_{i=k-\left[\tau_{1}\right]}^{k-1} b_{1}(i) \leq u_{1}\left(k-\left[\tau_{1}\right]\right)
$$


8 Harmless delays in a discrete periodic model

hence

$$
\begin{aligned}
N_{1}\left(k-\left[\tau_{1}\right]\right) & =\exp \left\{u_{1}\left(k-\left[\tau_{1}\right]\right)\right\} \geq \exp \left\{u_{1}(k)-\sum_{i=k-\left[\tau_{1}\right]}^{k-1} b_{1}(i)\right\} \\
& =N_{1}(k) \exp \left\{-\sum_{i=k-\left[\tau_{1}\right]}^{k-1} b_{1}(i)\right\} .
\end{aligned}
$$

Therefore

$$
N_{1}(k+1) \leq N_{1}(k) \exp \left\{b_{1}(k)-a_{1}(k) N_{1}(k) \exp \left\{-\sum_{i=k-\left[\tau_{1}\right]}^{k-1} b_{1}(i)\right\}\right\} .
$$

Consider the following auxiliary equation:

$$
z(k+1)=z(k) \exp \left\{b_{1}(k)-a_{1}(k) z(k) \exp \left\{-\sum_{i=k-\left[\tau_{1}\right]}^{k-1} b_{1}(i)\right\}\right\} .
$$

By Lemma 2.2, (2.25) has at least one positive $\omega$-periodic solution, denote it as $z^{*}(k)$, we have

$$
z^{*}(k) \leq \frac{\bar{b}_{1}}{\left(a_{1}(k) \exp \left\{-\sum_{i=k-\left[\tau_{1}\right]}^{k-1} b_{1}(i)\right\}\right)} \exp \left\{\bar{b}_{1}+\overline{\left|b_{1}\right|}\right\}:=H_{1}
$$

Let

$$
z^{*}(k)=\exp \left\{u_{2}(k)\right\}
$$

then

$$
\begin{aligned}
& u_{1}(k+1)-u_{1}(k) \leq b_{1}(k)-a_{1}(k) \exp \left\{u_{1}(k)\right\} \exp \left\{-\sum_{i=k-\left[\tau_{1}\right]}^{k-1} b_{1}(i)\right\}, \\
& u_{2}(k+1)-u_{2}(k)=b_{1}(k)-a_{1}(k) \exp \left\{u_{2}(k)\right\} \exp \left\{-\sum_{i=k-\left[\tau_{1}\right]}^{k-1} b_{1}(i)\right\} .
\end{aligned}
$$

Making the transformation $u(k)=u_{1}(k)-u_{2}(k)$, we can obtain

$$
u(k+1)-u(k) \leq-a_{1}(k)[\exp \{u(k)\}-1] \exp \left\{u_{2}(k)\right\} \exp \left\{-\sum_{i=k-\left[\tau_{1}\right]}^{k-1} b_{1}(i)\right\} .
$$

Now we divide the proof into two cases according to the oscillating property of $u(k)$. First we assume that $u(k)$ does not oscillate about zero, then $u(k)$ will be either eventually positive or eventually negative. If the latter holds, that is, $u_{1}(k)<u_{2}(k)$, we have

$$
N_{1}(k)<z^{*}(k) \leq\left(z^{*}(k)\right)^{M} .
$$


Whereas if the former holds, then by (2.29), we know $u(k+1)<u(k)$, which means that $u(k)$ is eventually decreasing, also in terms of its positivity, we know that $\lim _{k \rightarrow \infty} u(k)$ exists. Then (2.29) yields $\lim _{k \rightarrow \infty} u(k)=0$, which leads to

$$
\limsup _{k \rightarrow \infty} N_{1}(k) \leq\left(z^{*}(k)\right)^{M} .
$$

Now we assume that $u(k)$ oscillates about zero, by (2.29), we know that $u(k)>0$ implies $u(k+1) \leq u(k)$. Thus, if we let $\left\{u\left(k_{l}\right)\right\}$ be a subsequence of $\{u(k)\}$, where $u\left(k_{l}\right)$ is the first element of the positive semicycle of $\{u(k)\}$, then $\limsup _{k \rightarrow \infty} u(k)=\limsup _{l \rightarrow \infty} u\left(k_{l}\right)$. Combining

$$
u\left(k_{l}\right) \leq u\left(k_{l}-1\right)-a_{1}\left(k_{l}-1\right)\left[\exp \left\{u\left(k_{l}-1\right)\right\}-1\right] \exp \left\{u_{2}\left(k_{l}-1\right)\right\} \exp \left\{-\sum_{i=k_{l}-1-\left[\tau_{1}\right]}^{k_{l}-2} b_{1}(i)\right\}
$$

with $u\left(k_{l}-1\right)<0$, we know

$$
\begin{aligned}
u\left(k_{l} v\right) & \leq a_{1}\left(k_{l}-1\right)\left[\exp \left\{u\left(k_{l}-1\right)\right\}-1\right] \exp \left\{u_{2}\left(k_{l}-1\right)\right\} \exp \left\{-\sum_{i=k_{l}-1-\left[\tau_{1}\right]}^{k_{l}-2} b_{1}(i)\right\} \\
& \leq a_{1}\left(k_{l}-1\right) \exp \left\{u_{2}\left(k_{l}-1\right)\right\} \exp \left\{-\sum_{i=k_{l}-1-\left[\tau_{1}\right]}^{k_{l}-2} b_{1}(i)\right\} \\
& \leq\left(a_{1}\left(k_{l}-1\right) \exp \left\{u_{2}\left(k_{l}-1\right)\right\} \exp \left\{-\sum_{i=k_{l}-1-\left[\tau_{1}\right]}^{k_{l}-2} b_{1}(i)\right\}\right)^{M} .
\end{aligned}
$$

Therefore

$$
\limsup _{l \rightarrow \infty} u\left(k_{l}\right) \leq\left(a_{1}\left(k_{l}-1\right) \exp \left\{u_{2}\left(k_{l}-1\right)\right\} \exp \left\{-\sum_{i=k_{l}-1-\left[\tau_{1}\right]}^{k_{l}-2} b_{1}(i)\right\}\right)^{M} .
$$

By the medium of (2.27), we have $\limsup _{k \rightarrow \infty} N_{1}(k) \leq K_{1}$, where

$$
K_{1}=H_{1} \exp \left\{\left(a_{1}(k) H_{1} \exp \left\{-\sum_{i=k-\left[\tau_{1}\right]}^{k-1} b_{1}(i)\right\}\right)^{M}\right\} .
$$

Proposition 2.4. Under the condition (H1), there exists a positive constant $k_{1}$ such that $\liminf _{k \rightarrow \infty} N_{1}(k) \geq k_{1}$.

Proof. Given any positive solution $\left(N_{1}(k), N_{2}(k)\right)$ of (1.8), from the first equation of (1.8), we have

$$
N_{1}(k+1) \geq N_{1}(k) \exp \left\{b_{1}(k)-\frac{\alpha_{1}(k)}{m(k)}-a_{1}(k) N_{1}\left(k-\left[\tau_{1}\right]\right)\right\} .
$$


10 Harmless delays in a discrete periodic model

Set $N_{1}(k)=\exp \left\{u_{1}(k)\right\}$, then

$$
u_{1}(k+1)-u_{1}(k) \geq b_{1}(k)-\frac{\alpha_{1}(k)}{m(k)}-a_{1}(k) \exp \left\{u_{1}\left(k-\left[\tau_{1}\right]\right)\right\}
$$

which yields

$$
\sum_{i=k-\left[\tau_{1}\right]}^{k-1}\left(u_{1}(i+1)-u_{1}(i)\right) \geq \sum_{i=k-\left[\tau_{1}\right]}^{k-1}\left(b_{1}(i)-\frac{\alpha_{1}(i)}{m(i)}-a_{1}(i) K_{1}\right),
$$

that is,

$$
u_{1}\left(k-\left[\tau_{1}\right]\right) \leq u_{1}(k)-\sum_{i=k-\left[\tau_{1}\right]}^{k-1}\left(b_{1}(i)-\frac{\alpha_{1}(i)}{m(i)}-a_{1}(i) K_{1}\right)
$$

thus

$$
\begin{aligned}
N_{1}\left(k-\left[\tau_{1}\right]\right) & =\exp \left\{u_{1}\left(k-\left[\tau_{1}\right]\right)\right\} \\
& \leq \exp \left\{u_{1}(k)-\sum_{i=k-\left[\tau_{1}\right]}^{k-1}\left(b_{1}(i)-\frac{\alpha_{1}(i)}{m(i)}-a_{1}(i) K_{1}\right)\right\} \\
& =N_{1}(k) \exp \left\{-\sum_{i=k-\left[\tau_{1}\right]}^{k-1}\left(b_{1}(i)-\frac{\alpha_{1}(i)}{m(i)}-a_{1}(i) K_{1}\right)\right\} .
\end{aligned}
$$

Therefore

$$
\begin{aligned}
N_{1}(k+1) \geq N_{1}(k) \exp \{ & b_{1}(k)-\frac{\alpha_{1}(k)}{m(k)} \\
& \left.-a_{1}(k) N_{1}(k) \exp \left\{-\sum_{i=k-\left[\tau_{1}\right]}^{k-1}\left(b_{1}(i)-\frac{\alpha_{1}(i)}{m(i)}-a_{1}(i) K_{1}\right)\right\}\right\} .
\end{aligned}
$$

Consider the following auxiliary equation:

$$
z(k+1)=z(k) \exp \left\{b_{1}(k)-\frac{\alpha_{1}(k)}{m(k)}-a_{1}(k) z(k) \exp \left\{-\sum_{i=k-\left[\tau_{1}\right]}^{k-1}\left(b_{1}(i)-\frac{\alpha_{1}(i)}{m(i)}-a_{1}(i) K_{1}\right)\right\}\right\} .
$$

By Lemma 2.2 and (H1), (2.42) has at least one positive $\omega$-periodic solution, denoted as $z_{1}^{*}(k)$, then

$$
\begin{aligned}
& z_{1}^{*}(k) \geq \frac{\bar{b}_{1}-\overline{\left(\alpha_{1} / m\right)}}{\left(a_{1}(k) \exp \left\{-\sum_{i=k-\left[\tau_{1}\right]}^{k-1}\left(b_{1}(i)-\alpha_{1}(i) / m(i)-a_{1}(i) K_{1}\right)\right\}\right)} \\
& \times \exp \left\{-\bar{b}_{1}+\overline{\left(\frac{\alpha_{1}}{m}\right)}-\overline{\left|b_{1}(k)-\frac{\alpha_{1}(k)}{m(k)}\right|}\right\}:=H_{2} .
\end{aligned}
$$


Now make the change of variables:

$$
z_{1}^{*}(k)=\exp \left\{u_{2}(k)\right\}
$$

then

$$
\begin{aligned}
& u_{1}(k+1)-u_{1}(k) \geq b_{1}(k)-\frac{\alpha_{1}(k)}{m(k)} \\
&-a_{1}(k) \exp \left\{u_{1}(k)\right\} \exp \left\{-\sum_{i=k-\left[\tau_{1}\right]}^{k-1}\left(b_{1}(i)-\frac{\alpha_{1}(i)}{m(k)}-a_{1}(i) K_{1}\right)\right\}, \\
& \begin{aligned}
u_{2}(k+1)-u_{2}(k)= & b_{1}(k)-\frac{\alpha_{1}(k)}{m(k)} \\
& -a_{1}(k) \exp \left\{u_{2}(k)\right\} \exp \left\{-\sum_{i=k-\left[\tau_{1}\right]}^{k-1}\left(b_{1}(i)-\frac{\alpha_{1}(i)}{m(i)}-a_{1}(i) K_{1}\right)\right\} .
\end{aligned}
\end{aligned}
$$

Denote $u(k)=u_{1}(k)-u_{2}(k)$, we have

$$
\begin{aligned}
u(k+1)-u(k) \geq & -a_{1}(k)[\exp \{u(k)\}-1] \exp \left\{u_{2}(k)\right\} \\
& \times \exp \left\{-\sum_{i=k-\left[\tau_{1}\right]}^{k-1}\left(b_{1}(i)-\frac{\alpha_{1}(i)}{m(i)}-a_{1}(i) K_{1}\right)\right\} .
\end{aligned}
$$

If $u(k)$ does not oscillate, then by a similar analysis as that in Proposition 2.3, we have

$$
\liminf _{k \rightarrow \infty} N_{1}(k) \geq\left(z_{1}^{*}(k)\right)^{L}
$$

Whereas if $u(k)$ oscillates about zero, by (2.46), we know that if $u(k)<0$, then $u(k+$ $1) \geq u(k)$. Thus, if we denote $\left\{u\left(k_{l}\right)\right\}$ as a subsequence of $\{u(k)\}$, where $u\left(k_{l}\right)$ is the first element of the negative semicycle of $\{u(k)\}$, then $\liminf _{k \rightarrow \infty} u(k)=\liminf _{l \rightarrow \infty} u\left(k_{l}\right)$. On the other hand, from

$$
\begin{aligned}
u\left(k_{l}\right) \geq & u\left(k_{l}-1\right)-a_{1}\left(k_{l}-1\right)\left[\exp \left\{u\left(k_{l}-1\right)\right\}-1\right] \exp \left\{u_{2}\left(k_{l}-1\right)\right\} \\
& \times \exp \left\{-\sum_{i=k_{l}-1-\left[\tau_{1}\right]}^{k_{l}-2}\left(b_{1}(i)-\frac{\alpha_{1}(i)}{m(i)}-a_{1}(i) K_{1}\right)\right\},
\end{aligned}
$$


12 Harmless delays in a discrete periodic model

and $u\left(k_{l}-1\right)>0$, we know

$$
\begin{aligned}
u\left(k_{l}\right) \geq & a_{1}\left(k_{l}-1\right)\left[1-\exp \left\{u\left(k_{l}-1\right)\right\}\right] \exp \left\{u_{2}\left(k_{l}-1\right)\right\} \\
& \times \exp \left\{-\sum_{i=k_{l}-1-\left[\tau_{1}\right]}^{k_{l}-2}\left(b_{1}(i)-\frac{\alpha_{1}(i)}{m(i)}-a_{1}(i) K_{1}\right)\right\} \\
\geq & -a_{1}\left(k_{l}-1\right) \exp \left\{u_{1}\left(k_{l}-1\right)\right\} \\
& \times \exp \left\{-\sum_{i=k_{l}-1-\left[\tau_{1}\right]}^{k_{l}-2}\left(b_{1}(i)-\frac{\alpha_{1}(i)}{m(i)}-a_{1}(i) K_{1}\right)\right\} \\
\geq & \left(-K_{1} a_{1}\left(k_{l}-1\right) \exp \left\{-\sum_{i=k_{l}-1-\left[\tau_{1}\right]}^{k_{l}-2}\left(b_{1}(i)-\frac{\alpha_{1}(i)}{m(i)}-a_{1}(i) K_{1}\right)\right\}\right)^{L} .
\end{aligned}
$$

Therefore

$$
\liminf _{l \rightarrow \infty} u\left(k_{l}\right) \geq\left(-K_{1} a_{1}\left(k_{l}-1\right) \exp \left\{-\sum_{i=k_{l}-1-\left[\tau_{1}\right]}^{k_{l}-2}\left(b_{1}(i)-\frac{\alpha_{1}(i)}{m(i)}-a_{1}(i) K_{1}\right)\right\}\right)^{L} .
$$

By the medium of (2.44), we have

$$
\begin{aligned}
& \liminf _{k \rightarrow \infty} N_{1}(k) \\
& \quad \geq\left(z_{1}^{*}(k)\right)^{L} \exp \left\{\left(-K_{1} a_{1}(k) \exp \left\{-\sum_{i=k-\left[\tau_{1}\right]}^{k-1}\left(b_{1}(i)-\frac{\alpha_{1}(i)}{m(i)}-a_{1}(i) K_{1}\right)\right\}\right)^{L}\right\} ;
\end{aligned}
$$

hence $\liminf _{k \rightarrow \infty} N_{1}(k) \geq k_{1}$, where

$$
k_{1}=H_{2} \exp \left\{\left(-K_{1} a_{1}(k) \exp \left\{-\sum_{i=k-\left[\tau_{1}\right]}^{k-1}\left(b_{1}(i)-\frac{\alpha_{1}(i)}{m(i)}-a_{1}(i) K_{1}\right)\right\}\right)^{L}\right\} .
$$

Proposition 2.5. If (H2) holds, then there exists a positive constant $K_{2}$ such that

$$
\limsup _{k \rightarrow \infty} N_{2}(k) \leq K_{2}
$$


Proof. Given any positive solution $\left(N_{1}(k), N_{2}(k)\right)$ of $(1.8)$, from the second equation of (1.8), we have

$$
\begin{aligned}
N_{2}(k+1) & =N_{2}(k) \exp \left\{-b_{2}(k)+\frac{\alpha_{2}(k) N_{1}\left(k-\left[\tau_{2}\right]\right)}{N_{1}\left(k-\left[\tau_{2}\right]\right)+m(k) N_{2}\left(k-\left[\tau_{2}\right]\right)}\right\} \\
& \leq N_{2}(k) \exp \left\{-b_{2}(k)+\frac{\alpha_{2}(k) K_{1}}{K_{1}+m(k) N_{2}\left(k-\left[\tau_{2}\right]\right)}\right\} \\
& =N_{2}(k) \exp \left\{\alpha_{2}(k)-b_{2}(k)-\alpha_{2}(k)\left[\frac{m(k) N_{2}\left(k-\left[\tau_{2}\right]\right)}{K_{1}+m(k) N_{2}\left(k-\left[\tau_{2}\right]\right)}\right]\right\} .
\end{aligned}
$$

Set $N_{2}(k)=\exp \left\{u_{1}(k)\right\}$, then

$$
u_{1}(k+1)-u_{1}(k) \leq \alpha_{2}(k)-b_{2}(k)
$$

thus

$$
\sum_{i=k-\left[\tau_{2}\right]}^{k-1}\left(u_{1}(i+1)-u_{1}(i)\right) \leq \sum_{i=k-\left[\tau_{2}\right]}^{k-1}\left(\alpha_{2}(i)-b_{2}(i)\right)
$$

which is equivalent to

$$
u_{1}(k)-\sum_{i=k-\left[\tau_{2}\right]}^{k-1}\left(\alpha_{2}(i)-b_{2}(i)\right) \leq u_{1}\left(k-\left[\tau_{2}\right]\right) \text {; }
$$

hence

$$
\begin{aligned}
N_{2}\left(k-\left[\tau_{2}\right]\right) & =\exp \left\{u_{1}\left(k-\left[\tau_{2}\right]\right)\right\} \geq \exp \left\{u_{1}(k)-\sum_{i=k-\left[\tau_{2}\right]}^{k-1}\left(\alpha_{2}(i)-b_{2}(i)\right)\right\} \\
& =N_{2}(k) \exp \left\{-\sum_{i=k-\left[\tau_{2}\right]}^{k-1}\left(\alpha_{2}(i)-b_{2}(i)\right)\right\} .
\end{aligned}
$$

Therefore

$$
\begin{aligned}
N_{2}(k+1) \leq N_{2}(k) \exp \{ & \alpha_{2}(k)-b_{2}(k) \\
& \left.-\alpha_{2}(k)\left[\frac{m(k) N_{2}(k) \exp \left\{-\sum_{i=k-\left[\tau_{2}\right]}^{k-1}\left(\alpha_{2}(i)-b_{2}(i)\right)\right\}}{K_{1}+m(k) N_{2}(k) \exp \left\{-\sum_{i=k-\left[\tau_{2}\right]}^{k-1}\left(\alpha_{2}(i)-b_{2}(i)\right)\right\}}\right]\right\} .
\end{aligned}
$$

Here we use the monotonicity of the function $u /(a+u)$. 
14 Harmless delays in a discrete periodic model

Consider the following auxiliary equation:

$$
\begin{aligned}
z(k+1)=z(k) \exp \left\{\alpha_{2}(k)-b_{2}(k)\right. & \\
& \left.-\alpha_{2}(k)\left[\frac{m(k) z(k) \exp \left\{-\sum_{i=k-\left[\tau_{2}\right]}^{k-1}\left(\alpha_{2}(i)-b_{2}(i)\right)\right\}}{K_{1}+m(k) z(k) \exp \left\{-\sum_{i=k-\left[\tau_{2}\right]}^{k-1}\left(\alpha_{2}(i)-b_{2}(i)\right)\right\}}\right]\right\} .
\end{aligned}
$$

By the same method as that in [6], (2.60) has at least one positive $\omega$-periodic solution, denote it as $z_{2}^{*}(k)$, then through some simple calculations, we have

$$
\begin{aligned}
z_{2}^{*}(k) \leq & \frac{\left(\bar{\alpha}_{2}-\bar{b}_{2}\right) K_{1}}{\bar{b}_{2}\left(m(k) \exp \left\{-\sum_{i=k-\left[\tau_{2}\right]}^{k-1}\left(\alpha_{2}(i)-b_{2}(i)\right)\right\}\right)^{L}} \\
& \times \exp \left\{\bar{\alpha}_{2}-\bar{b}_{2}+\overline{\left|\alpha_{2}(k)-b_{2}(k)\right|}\right\}:=H_{3} .
\end{aligned}
$$

Let

$$
z_{2}^{*}(k)=\exp \left\{u_{2}(k)\right\}
$$

then

$$
\begin{aligned}
u_{1}(k+1)-u_{1}(k) \leq & \alpha_{2}(k)-b_{2}(k) \\
& -\alpha_{2}(k)\left[\frac{m(k) \exp \left\{u_{1}(k)\right\} \exp \left\{-\sum_{i=k-\left[\tau_{2}\right]}^{k-1}\left(\alpha_{2}(i)-b_{2}(i)\right)\right\}}{K_{1}+m(k) \exp \left\{u_{1}(k)\right\} \exp \left\{-\sum_{i=k-\left[\tau_{2}\right]}^{k-1}\left(\alpha_{2}(i)-b_{2}(i)\right)\right\}}\right], \\
u_{2}(k+1)-u_{2}(k)= & \alpha_{2}(k)-b_{2}(k) \\
& -\alpha_{2}(k)\left[\frac{m(k) \exp \left\{u_{2}(k)\right\} \exp \left\{-\sum_{i=k-\left[\tau_{2}\right]}^{k-1}\left(\alpha_{2}(i)-b_{2}(i)\right)\right\}}{K_{1}+m(k) \exp \left\{u_{2}(k)\right\} \exp \left\{-\sum_{i=k-\left[\tau_{2}\right]}^{k-1}\left(\alpha_{2}(i)-b_{2}(i)\right)\right\}}\right] .
\end{aligned}
$$

Denote $u(k)=u_{1}(k)-u_{2}(k)$, we have

$$
\begin{aligned}
u(k+1) & -u(k) \\
\leq & -\alpha_{2}(k)\left[\frac{m(k) K_{1} \exp \left\{u_{2}(k)\right\} \exp \left\{-\sum_{i=k-\left[\tau_{2}\right]}^{k-1}\left(\alpha_{2}(i)-b_{2}(i)\right)\right\}}{K_{1}+m(k) \exp \left\{u_{2}(k)\right\} \exp \left\{-\sum_{i=k-\left[\tau_{2}\right]}^{k-1}\left(\alpha_{2}(i)-b_{2}(i)\right)\right\}}\right] \\
& \times\left[\frac{\exp \{u(k)\}-1}{K_{1}+m(k) \exp \left\{u_{2}(k)\right\} \exp \{u(k)\} \exp \left\{-\sum_{i=k-\left[\tau_{2}\right]}^{k-1}\left(\alpha_{2}(i)-b_{2}(i)\right)\right\}}\right] .
\end{aligned}
$$


First we assume that $u(k)$ does not oscillate about zero, then $u(k)$ will be either eventually positive or eventually negative. If the latter holds, that is, $u_{1}(k)<u_{2}(k)$, we have

$$
N_{2}(k)<z_{2}^{*}(k) \leq\left(z_{2}^{*}(k)\right)^{M} .
$$

Whereas if the former holds, then by (2.64), we have $u(k+1)<u(k)$, which means that $u(k)$ is eventually decreasing, also in terms of its positivity, we obtain that $\lim _{k \rightarrow \infty} u(k)$ exists. Then (2.64) leads to $\lim _{k \rightarrow \infty} u(k)=0$, this implies

$$
\limsup _{k \rightarrow \infty} N_{2}(k) \leq\left(z_{2}^{*}(k)\right)^{M} .
$$

Now we assume that $u(k)$ oscillates about zero; in view of (2.64), we know that $u(k)>$ 0 implies $u(k+1) \leq u(k)$. Thus, if we let $\left\{u\left(k_{l}\right)\right\}$ be a subsequence of $\{u(k)\}$, where $u\left(k_{l}\right)$ is the first element of the positive semicycle of $\{u(k)\}$, then $\limsup _{k \rightarrow \infty} u(k)=$ $\lim \sup _{l \rightarrow \infty} u\left(k_{l}\right)$. Also, from

$$
\begin{aligned}
u\left(k_{l}\right) \leq & u\left(k_{l}-1\right) \\
& -\alpha_{2}\left(k_{l}-1\right)\left[\frac{m\left(k_{l}-1\right) K_{1} \exp \left\{u_{2}\left(k_{l}-1\right)\right\} \exp \left\{-\sum_{i=k_{l}-1-\left[\tau_{2}\right]}^{k_{l}-2}\left(\alpha_{2}(i)-b_{2}(i)\right)\right\}}{K_{1}+m\left(k_{l}-1\right) \exp \left\{u_{2}\left(k_{l}-1\right)\right\} \exp \left\{-\sum_{i=k_{l}-1-\left[\tau_{2}\right]}^{k_{l}-2}\left(\alpha_{2}(i)-b_{2}(i)\right)\right\}}\right] \\
& \times\left[\frac{\exp \left\{u\left(k_{l}-1\right)\right\}-1}{K_{1}+m\left(k_{l}-1\right) \exp \left\{u_{2}\left(k_{l}-1\right)\right\} \exp \left\{u\left(k_{l}-1\right)\right\} \exp \left\{-\sum_{i=k_{l}-1-\left[\tau_{2}\right]}^{k_{l}-2}\left(\alpha_{2}(i)-b_{2}(i)\right)\right\}}\right],
\end{aligned}
$$

and $u\left(k_{l}-1\right)<0$, we know

$$
\begin{aligned}
u\left(k_{l}\right) \leq & \alpha_{2}\left(k_{l}-1\right) \\
& \times\left[\frac{m\left(k_{l}-1\right) K_{1} \exp \left\{u_{2}\left(k_{l}-1\right)\right\} \exp \left\{-\sum_{i=k_{l}-1-\left[\tau_{2}\right]}^{k_{l}-2}\left(\alpha_{2}(i)-b_{2}(i)\right)\right\}}{K_{1}+m\left(k_{l}-1\right) \exp \left\{u_{2}\left(k_{l}-1\right)\right\} \exp \left\{-\sum_{i=k_{l}-1-\left[\tau_{2}\right]}^{k_{l}-2}\left(\alpha_{2}(i)-b_{2}(i)\right)\right\}}\right] \\
& \times\left[\frac{1-\exp \left\{u\left(k_{l}-1\right)\right\}}{K_{1}+m\left(k_{l}-1\right) \exp \left\{u_{2}\left(k_{l}-1\right)\right\} \exp \left\{u\left(k_{l}-1\right)\right\} \exp \left\{-\sum_{i=k_{l}-1-\left[\tau_{2}\right]}^{k_{l}-2}\left(\alpha_{2}(i)-b_{2}(i)\right)\right\}}\right] .
\end{aligned}
$$

Consider the function

$$
g(x)=\frac{1-x}{p+q x}, \quad p>0, q>0,0 \leq x \leq 1 .
$$

It is easy to show that $g(x)$ has the property $g(x) \leq g(0)$. Therefore (2.68) yields

$$
u\left(k_{l}\right) \leq \frac{\alpha_{2}\left(k_{l}-1\right) m\left(k_{l}-1\right) \exp \left\{u_{2}\left(k_{l}-1\right)\right\} \exp \left\{-\sum_{i=k_{l}-1-\left[\tau_{2}\right]}^{k_{l}-2}\left(\alpha_{2}(i)-b_{2}(i)\right)\right\}}{K_{1}+m\left(k_{l}-1\right)\left\{u_{2}\left(k_{l}-1\right)\right\} \exp \left\{-\sum_{i=k_{l}-1-\left[\tau_{2}\right]}^{k_{l}-2}\left(\alpha_{2}(i)-b_{2}(i)\right)\right\}},
$$


16 Harmless delays in a discrete periodic model

that is,

$$
\begin{aligned}
& \limsup _{l \rightarrow \infty} u\left(k_{l}\right) \\
& \quad \leq\left(\frac{\alpha_{2}\left(k_{l}-1\right) m\left(k_{l}-1\right) \exp \left\{u_{2}\left(k_{l}-1\right)\right\} \exp \left\{-\sum_{i=k_{l}-1-\left[\tau_{2}\right]}^{k_{l}-2}\left(\alpha_{2}(i)-b_{2}(i)\right)\right\}}{K_{1}+m\left(k_{l}-1\right) \exp \left\{u_{2}\left(k_{l}-1\right)\right\} \exp \left\{-\sum_{i=k_{l}-1-\left[\tau_{2}\right]}^{k_{l}-2}\left(\alpha_{2}(i)-b_{2}(i)\right)\right\}}\right)^{M} \\
& \quad \leq\left(\alpha_{2}\left(k_{l}-1\right)\right)^{M} .
\end{aligned}
$$

By the medium of (2.27), we have $\limsup _{k \rightarrow \infty} N_{2}(k) \leq K_{2}$, where

$$
K_{2}=H_{3} \exp \left\{\left(\alpha_{2}(k)\right)^{M}\right\}
$$

Proposition 2.6. Under the conditions (H1) and (H2), there exists a positive constant $k_{2}$ such that $\liminf _{k \rightarrow+\infty} N_{2}(k) \geq k_{2}$.

Proof. Given any positive solution $\left(N_{1}(k), N_{2}(k)\right)$ of $(1.8)$, from the second equation of (1.8), we have

$$
\begin{aligned}
N_{2}(k+1) & \\
& =N_{2}(k) \exp \left\{-b_{2}(k)+\frac{\alpha_{2}(k) N_{1}\left(k-\left[\tau_{2}\right]\right)}{N_{1}\left(k-\left[\tau_{2}\right]\right)+m(k) N_{2}\left(k-\left[\tau_{2}\right]\right)}\right\} \\
& =N_{2}(k) \exp \left\{\alpha_{2}(k)-b_{2}(k)+\alpha_{2}(k)\left[\frac{N_{1}\left(k-\left[\tau_{2}\right]\right)}{N_{1}\left(k-\left[\tau_{2}\right]\right)+m(k) N_{2}\left(k-\left[\tau_{2}\right]\right)}-1\right]\right\} \\
& =N_{2}(k) \exp \left\{\alpha_{2}(k)-b_{2}(k)-\alpha_{2}(k)\left[\frac{m(k) N_{2}\left(k-\left[\tau_{2}\right]\right)}{N_{1}\left(k-\left[\tau_{2}\right]\right)+m(k) N_{2}\left(k-\left[\tau_{2}\right]\right)}\right]\right\},
\end{aligned}
$$

then

$$
N_{2}(k+1) \geq N_{2}(k) \exp \left\{\alpha_{2}(k)-b_{2}(k)-\frac{\alpha_{2}(k) m(k) N_{2}\left(k-\left[\tau_{2}\right]\right)}{k_{1}}\right\} .
$$

In view of

$$
\begin{aligned}
N_{2}(k+1) & =N_{2}(k) \exp \left\{-b_{2}(k)+\frac{\alpha_{2}(k) N_{1}\left(k-\left[\tau_{2}\right]\right)}{N_{1}\left(k-\left[\tau_{2}\right]\right)+m(k) N_{2}\left(k-\left[\tau_{2}\right]\right)}\right\} \\
& \geq N_{2}(k) \exp \left\{-b_{2}(k)\right\},
\end{aligned}
$$


if we let $N_{2}(k)=\exp \left\{u_{1}(k)\right\}$, then we can obtain $u_{1}(k+1)-u_{1}(k) \geq-b_{2}(k)$. Thus

$$
\sum_{i=k-\left[\tau_{2}\right]}^{k-1}\left(u_{1}(i+1)-u_{1}(i)\right) \geq \sum_{i=k-\left[\tau_{2}\right]}^{k-1}\left(-b_{2}(i)\right)
$$

that is,

$$
u_{1}\left(k-\left[\tau_{2}\right]\right) \leq u_{1}(k)+\sum_{i=k-\left[\tau_{2}\right]}^{k-1} b_{2}(i)
$$

hence

$$
\begin{aligned}
N_{2}\left(k-\left[\tau_{2}\right]\right) & =\exp \left\{u_{1}\left(k-\left[\tau_{2}\right]\right)\right\} \leq \exp \left\{u_{1}(k)+\sum_{i=k-\left[\tau_{2}\right]}^{k-1} b_{2}(i)\right\} \\
& =N_{2}(k) \exp \left\{\sum_{i=k-\left[\tau_{2}\right]}^{k-1} b_{2}(i)\right\} .
\end{aligned}
$$

Therefore from (2.74), we have

$$
N_{2}(k+1) \geq N_{2}(k) \exp \left\{\alpha_{2}(k)-b_{2}(k)-\frac{\alpha_{2}(k) m(k)}{k_{1}} N_{2}(k) \exp \left\{\sum_{i=k-\left[\tau_{2}\right]}^{k-1} b_{2}(i)\right\}\right\} .
$$

Consider the auxiliary equation

$$
z(k+1)=z(k) \exp \left\{\alpha_{2}(k)-b_{2}(k)-\frac{\alpha_{2}(k) m(k)}{k_{1}} z(k) \exp \left\{\sum_{i=k-\left[\tau_{2}\right]}^{k-1} b_{2}(i)\right\}\right\} .
$$

By Lemma 2.2 and (H2), (2.80) has at least one positive $\omega$-periodic solution, denoted as $z_{3}^{*}(k)$, then

$$
\begin{aligned}
z_{3}^{*}(k) \geq & \frac{\left(\bar{\alpha}_{2}-\bar{b}_{2}\right) k_{1}}{\left(\alpha_{2}(k) m(k) \exp \left\{\sum_{i=k-\left[\tau_{2}\right]}^{k-1} b_{2}(i)\right\}\right)} \\
& \times \exp \left\{-\bar{\alpha}_{2}+\bar{b}_{2}-\overline{\left|\alpha_{2}(k)-b_{2}(k)\right|}\right\}:=H_{4} .
\end{aligned}
$$

If we set

$$
z_{3}^{*}(k)=\exp \left\{u_{2}(k)\right\}
$$


18 Harmless delays in a discrete periodic model

then

$$
\begin{aligned}
& u_{1}(k+1)-u_{1}(k) \geq \alpha_{2}(k)-b_{2}(k)-\frac{\alpha_{2}(k) m(k)}{k_{1}} \exp \left\{u_{1}(k)\right\} \exp \left\{\sum_{i=k-\left[\tau_{2}\right]}^{k-1} b_{2}(i)\right\}, \\
& u_{2}(k+1)-u_{2}(k)=\alpha_{2}(k)-b_{2}(k)-\frac{\alpha_{2}(k) m(k)}{k_{1}} \exp \left\{u_{2}(k)\right\} \exp \left\{\sum_{i=k-\left[\tau_{2}\right]}^{k-1} b_{2}(i)\right\} .
\end{aligned}
$$

And let $u(k)=u_{1}(k)-u_{2}(k)$, we have

$$
\begin{aligned}
& u(k+1)-u(k) \\
& \quad \geq-\frac{\alpha_{2}(k) m(k)}{k_{1}}[\exp \{u(k)\}-1] \exp \left\{u_{2}(k)\right\} \exp \left\{\sum_{i=k-\left[\tau_{2}\right]}^{k-1} b_{2}(i)\right\} .
\end{aligned}
$$

If $u(k)$ does not oscillate, then by a similar analysis as that in Proposition 2.3, we have

$$
\liminf _{k \rightarrow \infty} N_{2}(k) \geq\left(z_{3}^{*}(k)\right)^{L}
$$

Whereas if $u(k)$ oscillates about zero, by (2.84), we know that $u(k)<0$ implies $u(k+$ $1) \geq u(k)$. Thus, if we denote $\left\{u\left(k_{l}\right)\right\}$ as a subsequence of $\{u(k)\}$, where $u\left(k_{l}\right)$ is the first element of the negative semicycle of $\{u(k)\}$, then $\liminf _{k \rightarrow \infty} u(k)=\liminf _{l \rightarrow \infty} u\left(k_{l}\right)$. On the other hand, the combination of

$$
\begin{aligned}
u\left(k_{l}\right) \geq & u\left(k_{l}-1\right)-\frac{\alpha_{2}\left(k_{l}-1\right) m\left(k_{l}-1\right)}{k_{1}} \\
& \times\left[\exp \left\{u\left(k_{l}-1\right)\right\}-1\right] \exp \left\{u_{2}\left(k_{l}-1\right)\right\} \exp \left\{\sum_{i=k_{l}-1-\left[\tau_{2}\right]}^{k_{l}-2} b_{2}(i)\right\}
\end{aligned}
$$

and $u\left(k_{l}-1\right)>0$ gives

$$
\begin{aligned}
u\left(k_{l}\right) \geq & \frac{\alpha_{2}\left(k_{l}-1\right) m\left(k_{l}-1\right)}{k_{1}}\left[1-\exp \left\{u\left(k_{l}-1\right)\right\}\right] \exp \left\{u_{2}\left(k_{l}-1\right)\right\} \\
& \times \exp \left\{\sum_{i=k_{l}-1-\left[\tau_{2}\right]}^{k_{l}-2} b_{2}(i)\right\} \\
\geq & -\frac{\alpha_{2}\left(k_{l}-1\right) m\left(k_{l}-1\right)}{k_{1}} \exp \left\{u_{1}\left(k_{l}-1\right)\right\} \exp \left\{\sum_{i=k_{l}-1-\left[\tau_{2}\right]}^{k_{l}-2} b_{2}(i)\right\} \\
\geq & \left(-\frac{\alpha_{2}\left(k_{l}-1\right) m\left(k_{l}-1\right)}{k_{1}} K_{2} \exp \left\{\sum_{i=k_{l}-1-\left[\tau_{2}\right]}^{k_{l}-2} b_{2}(i)\right\}\right)^{L} .
\end{aligned}
$$


Therefore

$$
\begin{aligned}
\liminf _{l \rightarrow \infty} & u\left(k_{l}\right) \\
& \geq\left(-\frac{\alpha_{2}\left(k_{l}-1\right) m\left(k_{l}-1\right)}{k_{1}} K_{2} \exp \left\{\sum_{i=k_{l}-1-\left[\tau_{2}\right]}^{k_{l}-2} b_{2}(i)\right\}\right)^{L} .
\end{aligned}
$$

By the medium of (2.82), we have

$$
\begin{aligned}
\liminf _{k \rightarrow \infty} & N_{1}(k) \\
& \geq\left(z_{3}^{*}(k)\right)^{L} \exp \left\{\left(-\frac{\alpha_{2}(k) m(k)}{k_{1}} K_{2} \exp \left\{\sum_{i=k-\left[\tau_{2}\right]}^{k-1} b_{2}(i)\right\}\right)^{L}\right\} .
\end{aligned}
$$

Hence $\liminf _{k \rightarrow \infty} N_{1}(k) \geq k_{2}$, where

$$
k_{2}=H_{4} \exp \left\{\left(-\frac{\alpha_{2}(k) m(k)}{k_{1}} K_{2} \exp \left\{\sum_{i=k-\left[\tau_{2}\right]}^{k-1} b_{2}(i)\right\}\right)^{L}\right\} .
$$

Proof of Theorem 1.4. From Propositions 2.3-2.6, we can easily know that system (1.8) is permanent. The proof is complete.

We remark that, in the above discussions, we have obtained that under the conditions (H1) and (H2), system (1.8) has at least one periodic solutions and it is also permanent. Naturally we may conjecture whether the existence of positive periodic solutions of system (1.8) implies its permanence or the permanence of system (1.8) implies the existence of positive periodic solutions. This is a more challenging and interesting problem for future study.

\section{Acknowledgments}

The first author was supported by the Nature Science Foundation of Ludong University (24070301). The second author was supported by the NNSF of China (10571078).

\section{References}

[1] R. P. Agarwal, Difference Equations and Inequalities: Theory, Methods, and Applications, Monographs and Textbooks in Pure and Applied Mathematics, vol. 228, Marcel Dekker, New York, 2000.

[2] R. Arditi, N. Perrin, and H. Saiah, Functional response and heterogeneities: an experiment test with cladocerans, Oikos 60 (1991), 69-75.

[3] E. Beretta and Y. Kuang, Global analyses in some delayed ratio-dependent predator-prey systems, Nonlinear Analysis. Theory, Methods \& Applications 32 (1998), no. 3, 381-408.

[4] A. A. Berryman, The origins and evolution of predator-prey theory, Ecology 73 (1992), 1530-1535. 
[5] Y.-H. Fan, W.-T. Li, and L.-L. Wang, Periodic solutions of delayed ratio-dependent predator-prey models with monotonic or nonmonotonic functional responses, Nonlinear Analysis. Real World Applications 5 (2004), no. 2, 247-263.

[6] M. Fan and K. Wang, Periodic solutions of a discrete time nonautonomous ratio-dependent predator-prey system, Mathematical and Computer Modelling 35 (2002), no. 9-10, 951-961.

[7] H. I. Freedman, Deterministic Mathematical Models in Population Ecology, Monographs and Textbooks in Pure and Applied Mathematics, vol. 57, Marcel Dekker, New York, 1980.

[8] K. Gopalsamy, Stability and Oscillations in Delay Differential Equations of Population Dynamics, Mathematics and Its Applications, vol. 74, Kluwer Academic, Dordrecht, 1992.

[9] I. Hanski, The functional response of predators: worries about scale, Trends in Ecology \& Evolution 6 (1991), no. 5, 141-142.

[10] C. S. Holling, The functional response of predator to prey density and its role in mimicry and population regulation, Memoirs of Entomological Society of Canada 45 (1965), 1-60.

[11] S.-B. Hsu, T.-W. Hwang, and Y. Kuang, Global analysis of the Michaelis-Menten-type ratiodependent predator-prey system, Journal of Mathematical Biology 42 (2001), no. 6, 489-506.

[12] _ Rich dynamics of a ratio-dependent one-prey two-predators model, Journal of Mathematical Biology 43 (2001), no. 5, 377-396.

[13] H.-F. Huo and W.-T. Li, Permanence and global stability of positive solutions of a nonautonomous discrete ratio-dependent predator-prey model, Discrete Dynamics in Nature and Society 2005 (2005), no. 2, 135-144.

[14] C. Jost, O. Arino, and R. Arditi, About deterministic extinction in ratio-dependent predator-prey models, Bulletin of Mathematical Biology 61 (1999), no. 1, 19-32.

[15] V. L. Kocić and G. Ladas, Global Behavior of Nonlinear Difference Equations of Higher Order with Applications, Mathematics and Its Applications, vol. 256, Kluwer Academic, Dordrecht, 1993.

[16] Y. Kuang, Delay Differential Equations with Applications in Population Dynamics, Mathematics in Science and Engineering, vol. 191, Academic Press, Massachusetts, 1993.

[17] Y. Kuang and E. Beretta, Global qualitative analysis of a ratio-dependent predator-prey system, Journal of Mathematical Biology 36 (1998), no. 4, 389-406.

[18] W.-T. Li, Y.-H. Fan, and S. Ruan, Periodic solutions in a delayed predator-prey model with nonmonotonic functional response, submitted.

[19] R. M. May, Complexity and Stability in Model Ecosystems, Princeton University Press, New Jersey, 1973.

[20] _ Biological populations obeying difference equations: stable points, stable cycles, and chaos, Journal of Theoretical Biology 51 (1975), no. 2, 511-524.

[21] J. D. Murry, Mathematical Biology, Springer, New York, 1989.

[22] M. L. Rosenzweig, Paradox of enrichment: destabilization of exploitation ecosystem in ecological time, Science 171 (1971), 385-387.

[23] S. Ruan and D. Xiao, Global analysis in a predator-prey system with nonmonotonic functional response, SIAM Journal on Applied Mathematics 61 (2001), no. 4, 1445-1472.

[24] Y. Takeuchi, Global Dynamical Properties of Lotka-Volterra Systems, World Scientific, New Jersey, 1996.

[25] L.-L. Wang and W.-T. Li, Existence and global stability of positive periodic solutions of a predatorprey system with delays, Applied Mathematics and Computation 146 (2003), no. 1, 167-185.

[26] _ Periodic solutions and permanence for a delayed nonautonomous ratio-dependent predator-prey model with Holling type functional response, Journal of Computational and Applied Mathematics 162 (2004), no. 2, 341-357.

[27] D. Xiao and S. Ruan, Global dynamics of a ratio-dependent predator-prey system, Journal of Mathematical Biology 43 (2001), no. 3, 268-290.

[28] D. Xiao and Z. Zhang, On the uniqueness and nonexistence of limit cycles for predator-prey systems, Nonlinearity 16 (2003), no. 3, 1185-1201. 
[29] H. Zhu, S. A. Campbell, and G. S. K. Wolkowicz, Bifurcation analysis of a predator-prey system with nonmonotonic functional response, SIAM Journal on Applied Mathematics 63 (2002), no. 2, 636-682.

Yong-Hong Fan: School of Mathematics and Information, Ludong University, Yantai, Shandong 264025, China

E-mail address: fanyh02@st.lzu.edu.cn

Wan-Tong Li: School of Mathematics and Statistics, Lanzhou University, Lanzhou, Gansu 730000, China

E-mail address: wtli@lzu.edu.cn 


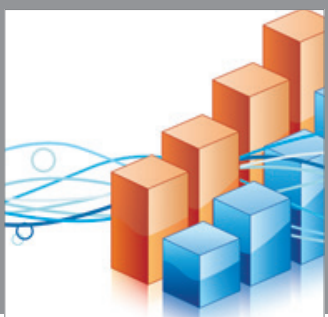

Advances in

Operations Research

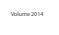

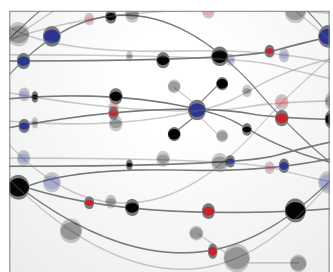

\section{The Scientific} World Journal
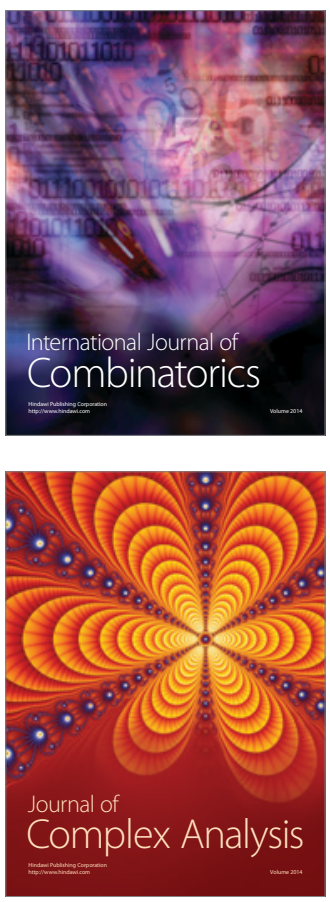

International Journal of

Mathematics and

Mathematical

Sciences
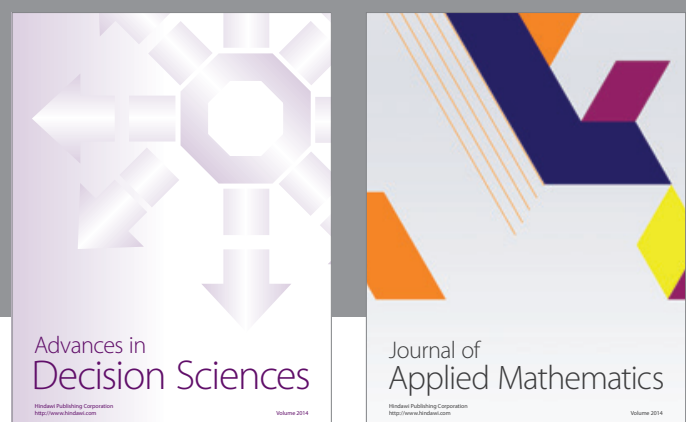

Journal of

Applied Mathematics
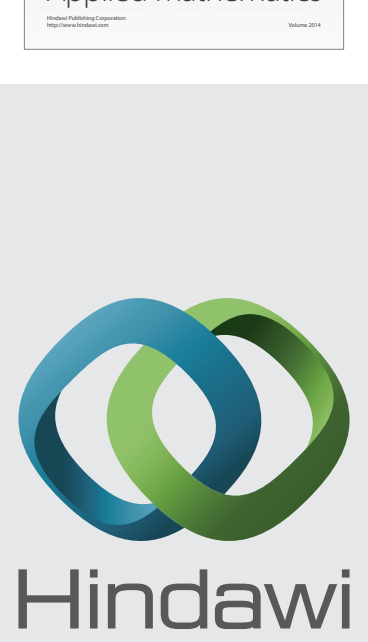

Submit your manuscripts at http://www.hindawi.com
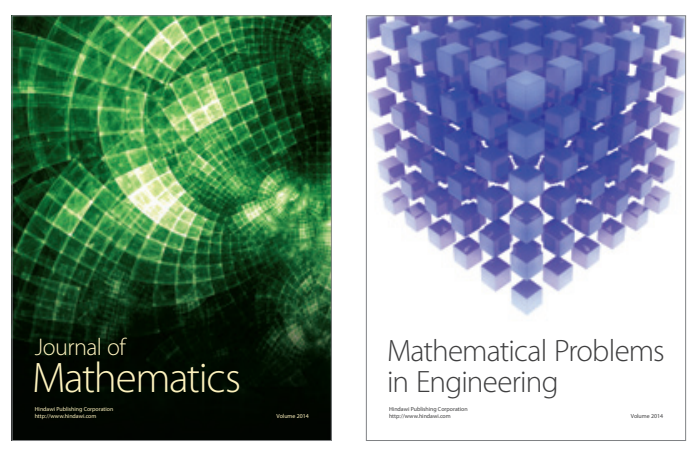

Mathematical Problems in Engineering
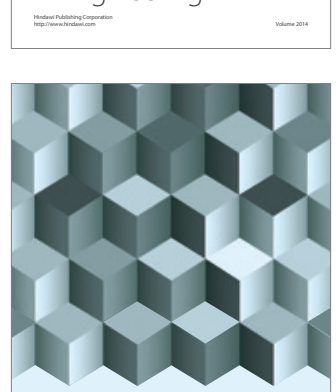

Journal of

Function Spaces
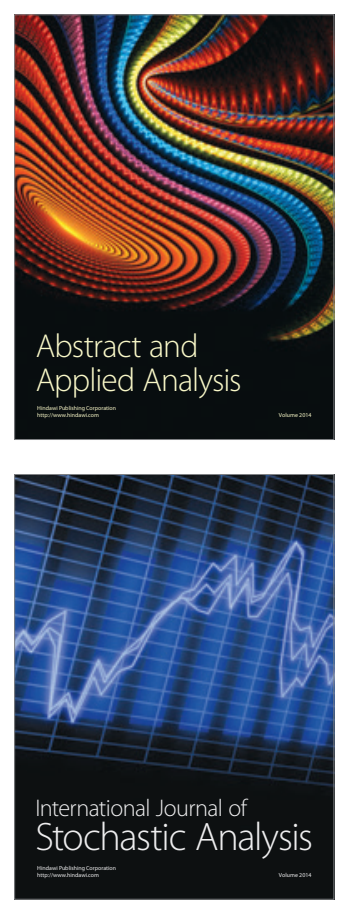

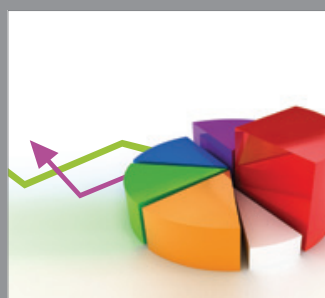

ournal of

Probability and Statistics

Promensencen
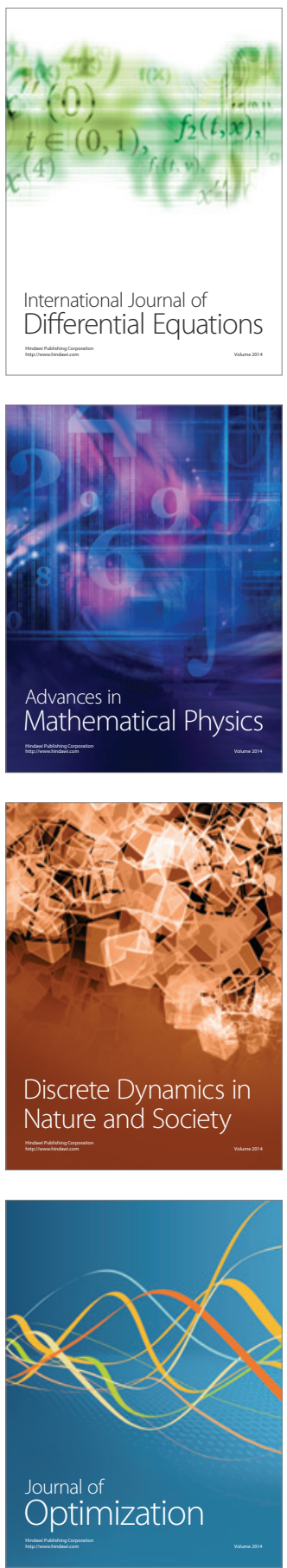